Research article Open Access

\title{
Analysis of dendritic cells in tumor-free and tumor-containing sentinel lymph nodes from patients with breast cancer
}

\author{
Nancy J Poindexter ${ }^{1}$, Aysegul Sahin ${ }^{2}$, Kelly K Hunt ${ }^{3}$ and Elizabeth A Grimm ${ }^{1}$
}

\author{
1Department of Bioimmunotherapy, The University of Texas MD Anderson Cancer Center, Houston, Texas, USA \\ 2Department of Pathology, The University of Texas MD Anderson Cancer Center, Houston, Texas, USA \\ ${ }^{3}$ Department of Surgical Oncology, The University of Texas MD Anderson Cancer Center, Houston, Texas, USA
}

Corresponding author: Nancy J Poindexter, npoindex@mdanderson.org

Received: 22 Jan 2004 Revisions requested: 4 Mar 2004 Revisions received: 29 Mar 2004 Accepted: 5 May 2004 Published: 4 Jun 2004

Breast Cancer Res 2004, 6:R408-R415 (DOI 10.1186/bcr808)

(c) 2004 Poindexter et al.; licensee BioMed Central Ltd. This is an Open Access article: verbatim copying and redistribution of this article are permitted in all media for any purpose, provided this notice is preserved along with the article's original URL.

\begin{abstract}
Introduction Sentinel lymph node (SLN) biopsy allows identification of the first lymph node into which a primary tumor drains. In breast cancer, identification of tumor cells in the SLNs is a predictor of the tumor's metastatic potential. In the present article, we tested the hypotheses that a positive immune response can occur in tumor-free SLNs and that the activation state of dendritic cells (DCs), the major antigen presenting cells within SLNs, predicts the immune status and metastatic potential of the tumor.
\end{abstract}

Methods Fifty paraffin-embedded SLN sections, 25 tumor-free and 25 tumor-containing, from patients with breast cancer were analyzed by immunohistochemistry to determine the immune maturation state of their DCs. In addition, 12 lymph nodes from noncancer-containing breasts were analyzed. Tissues were stained with antibodies against CD3, MHC class II, CD1a,
CD83, IL-10, and IL-12. Mature DCs were defined by CD83 expression and immature DCs by CD1a expression.

Results We found a trend toward higher numbers of mature CD83-positive DCs in tumor-free SLNs than in tumor-containing SLNs $(P=0.07)$. In addition, tumor-free SLNs were more likely to contain cells expressing IL-10 $(P=0.02)$ and, to a lesser extent, IL-12 $(P=0.12)$. In contrast, when all SLNs, both tumorfree and tumor-containing, were compared with uninvolved lymph nodes, the numbers of mature and immature DCs were similar.

Conclusions Our results suggest tumor-free SLNs are immunologically competent and potentially a site of tumorspecific T-cell activation, as evidenced by the presence of greater numbers of mature DCs and cytokine-producing cells in tumor-free SLNs.

Keywords: CD83, dendritic cells, IL-10, IL-12, sentinel lymph node

\section{Introduction}

Tumor-specific T-cell activation begins in the primary tumor when dendritic cells (DCs) encounter antigens in the form of apoptotic or necrotic tumor cells. The DCs engulf dying tumor cells and process their antigens into peptides that are presented in the context of MHC class I and class II molecules $[1,2]$. The function of a DC is highly influenced by its level of maturation. Immature DCs are capable of antigen uptake and processing but cannot, unless given the proper cytokine signals, present antigen to $T$ cells $[1,3,4]$. After receiving the correct cytokine signals, the mature, peptide-loaded DCs migrate from the tumor to the first draining lymph node, referred to as the sentinel lymph node (SLN). In the SLN, naïve T cells are activated by the peptide-loaded mature DCs. These T cells then undergo clonal expansion, gain effector function, and circulate back to the tumor, where their function is to lyse tumor cells. Evidence to support this process comes almost entirely from in vitro experiments [1-4].

SLN biopsy allows identification of the first lymph node into which a primary tumor drains. In breast cancer, identification of tumor cells in the SLNs is a predictor of the tumor's metastatic potential $[5,6]$. In the present study, we examined SLNs for evidence of immune activation by examining the maturation state of DCs within the SLNs. We defined mature DCs by their expression of the marker CD83 [7,8], while immature DCs were identified by their expression of the marker CD1a. We were interested in determining whether the maturation status of DCs in SLNs was 
associated with the tumor status of the SLN, so we compared DCs in tumor-free SLNs and in tumor-containing SLNs.

\section{Materials and methods Study population}

SLN tissues from women aged 26-87 years who had a SLN biopsy performed at The University of Texas MD Anderson Cancer Center between 1998 and 2001 were included in the study. Each of the patients had received a diagnosis of breast cancer and had undergone SLN biopsy as part of her surgical treatment. Paraffin-embedded SLN tissues from 50 patients, 25 with tumor-free SLNs and 25 with tumor-containing SLNs, were examined. The tumor status of the SLN was determined by $\mathrm{H}$ \& $\mathrm{E}$ and immunohistochemical staining. All samples were banked in the Breast Tumor Bank at The University of Texas MD Anderson Cancer Center.

The study population included six women who received chemotherapy prior to their SLN biopsy: four whose SLNs contained tumors and two whose SLNs were tumor-free. Twelve lymph nodes draining from the unaffected breast of women with breast cancer were similarly processed. All materials were collected under a protocol approved by the MD Anderson Cancer Center Institutional Review Board.

\section{Antibodies}

The following antibodies were used for immunohistochemical staining: anti-CD3 (clone PS1; BioGenex, San Ramon, CA, USA), anti-HLA class II (clone CR3/43; DAKO, Carpinteria, CA, USA), anti-CD83 (clone HB15A; Immunotech, Marseille, France), anti-CD1a (clone O10; Immunotech), anti-IL-10 (clone 23738.111; R \& D Systems, Minneapolis, MN, USA), and anti-IL-12 (clone 24910.1; R \& D Systems). Optimal concentrations were determined empirically, and all antibodies were tested for staining of cells in cytospin preparations of peripheral blood mononuclear cell (PBMC)-derived immature DCs (PBMCs stimulated with granulocyte-macrophage colony-stimulated factor and IL-4 for 6 days) or mature DCs (PBMCs stimulated with granulocyte-macrophage colony-stimulated factor and IL- 4 for 6 days followed by tumor necrosis factor alpha for 24 hours).

\section{Immunohistochemistry}

Tissue sections, $3 \mu \mathrm{m}$ thick, were cut from tissue blocks of formalin-fixed, paraffin-embedded SLNs. Immunocytochemical staining of deparaffinized, fixed slides was performed after antigen retrieval by microwave heating in citrate buffer. Slides were incubated with biotinylated goat anti-mouse lgG followed by avidin-biotin-peroxidase complex (Vectastain ABC Kit; Vector Laboratories, Berlingame, CA, USA). The peroxidase was developed by 3-amino-9ethylcarbazole-9 (AEC substrate kit; Vector Laboratories) and counterstained with Gill's hematoxylin (Vector Laboratories). Isotype-matched antibodies were used as negative controls. The positive control antibody for each group of slides was anti-CD3.

Because of the localized distribution of DCs within the SLNs, each slide was scored by counting positively stained cells in five high-powered fields (hpfs) under $\times 400$ magnification. Results were reported for each SLN as the mean \pm standard error of the mean for 5 hpfs. This method has been used by other workers when analyzing the expression of mature DCs in breast tissue [2,9]. All slides were counted without knowledge of the SLN status, and results were confirmed through a second reading by a breast cancer pathologist (AS).

\section{Statistical analysis}

All data sets were tested for normal distribution. Because most data groups were not normally distributed, all data groups were analyzed by the Wilcoxon rank sum test. $P<$ 0.05 was considered significant.

\section{Results \\ Clinicopathologic characteristics of the patient population}

Table 1 presents the clinicopathologic characteristics of the patients whose SLNs were included in this study. Patients ranged from 26 to 87 years of age at the time of diagnosis. No significant difference in median age distinguished the tumor-free SLN and the tumor-containing SLN groups (56 and 55 years, respectively). Additionally, we examined the estrogen receptor and progesterone receptor status, the size, grade, and the stage of disease of the tumors. There were no remarkable differences between the two groups of patients.

Comparison of mature and immature DCs within the SLN SLNs were examined for cells expressing CD83 and CD1a. Immature DCs, characterized by the expression of CD1a, were generally found in the paracortical areas of the SLNs. CD1a-expressing cells with typical DC morphology were found in 49 of 50 SLNs examined, with a median of 55 cells/hpf (range, 0-123 cells/hpf). Mature DCs expressing CD83 were found in 47 of 50 SLNs, with a median of 7 cells/hpf (range, 0-38 cells/hpf). Figure 1 shows the patterns of CD83 and CD1a expression in a tumor-free SLN and a tumor-containing SLN, respectively. At the magnification shown $(x 400)$, the different staining patterns are apparent, with the antibody to CD1a staining more dendriform cells than the CD83 antibody, which stains cells with much shorter dendrites. Another interpretation is that the CD83 antibody stains both membranous and cytoplasmic CD83, whereas the CD1a antibody stains only the membranous CD1a. Figure 2 shows sequential slides from the same paraffin block stained with the antibody to CD3, 


\begin{tabular}{|c|c|c|}
\hline & Tumor-free SLNs $(n=25)$ & Tumor-containing SLNs $(n=25)$ \\
\hline \multicolumn{3}{|l|}{ Patient age (years) } \\
\hline Median & 56 & 55 \\
\hline Range & $35-76$ & $26-87$ \\
\hline \multicolumn{3}{|l|}{ Tumor size $(\mathrm{cm})$} \\
\hline Median & 1.4 & 1.5 \\
\hline Range & $0.1-5$ & $0.6-4.5$ \\
\hline \multicolumn{3}{|l|}{ Steroid receptor status } \\
\hline Estrogen receptor-positive & $17(68 \%)^{a}$ & $19(76 \%)$ \\
\hline Estrogen receptor-negative & $4(16 \%)$ & $6(24 \%)$ \\
\hline Unknown & $4(16 \%)$ & 0 \\
\hline Progesterone receptor-positive & $15(60 \%)$ & $18(72 \%)$ \\
\hline Progesterone receptor-negative & $5(20 \%)$ & $7(28 \%)$ \\
\hline Unknown & $5(20 \%)$ & 0 \\
\hline \multicolumn{3}{|l|}{ Tumor grade } \\
\hline Grade I & $3(12 \%)$ & $3(12 \%)$ \\
\hline Grade II & $11(44 \%)$ & $12(48 \%)$ \\
\hline Grade III & $8(32 \%)$ & $8(32 \%)$ \\
\hline Unknown & $3(12 \%)$ & $2(8 \%)$ \\
\hline
\end{tabular}

a Data presented as number (percentage of total number of SLNs in each category).

MHC class II, CD1a, and CD83. These slides are representative of the SLNs identified as tumor-free. The CD83-positive cells were found surrounding the paracortical T-cell areas, usually near the sinuses of the SLNs. Although double staining was not performed, examination of sequential sections stained with anti-CD83 and antiMHC class II antibodies strongly suggested that the cells staining positively for CD83 were also positive for MHC class II.

When SLNs were compared by tumor burden, it was found that tumor-free SLNs contained a higher number of CD83positive cells than tumor-containing SLNs. The numbers of CD1a-positive cells within these two groups of SLNs were similar. Figure 3 shows the comparison between the numbers of CD1a and CD83 DCs in tumor-free and tumor-containing SLNs. There was a trend $(P=0.07)$ toward higher numbers of CD83-positive, mature DCs in tumor-free SLNs (median, 10.4 cells/hpf) than in tumor-containing SLNs (median, 4.5 cells/hpf). The numbers of CD1a-positive immature DCs did not differ between the tumor-free and tumor-containing SLNs $(P=0.93)$.

\section{Comparison of clinicopathologic features of the tumor and DCs in the SLN}

When tumors were analyzed based on hormone receptor status or tumor size, the numbers of CD83-expressing or CD1a-expressing cells in tumor-free and tumor-containing SLNs were not significantly different (data not shown). Notably, however, when tumors were sorted by nuclear grade and SLN status, we found that the number of CD1apositive, immunologically immature DCs was significantly greater $(P=0.01)$ in the tumor-containing SLNs draining from grade III tumors than in the tumor-free SLNs draining from grade III tumors (Table 2). These data suggest that the differentiation status of the tumor affected the maturation of the DCs within the tumor.

\section{Cytokine expression in the SLN}

Pivotal cytokines in the generation of a Th1 response versus a Th2 response are IL-10 and IL-12. DCs will respond to these cytokines and become polarized toward activating Th1 and Th2 [10]. We examined the SLNs for these two cytokines to determine whether there was an indication of this activation. An analysis of cytokine-expressing cells is 
Figure 1

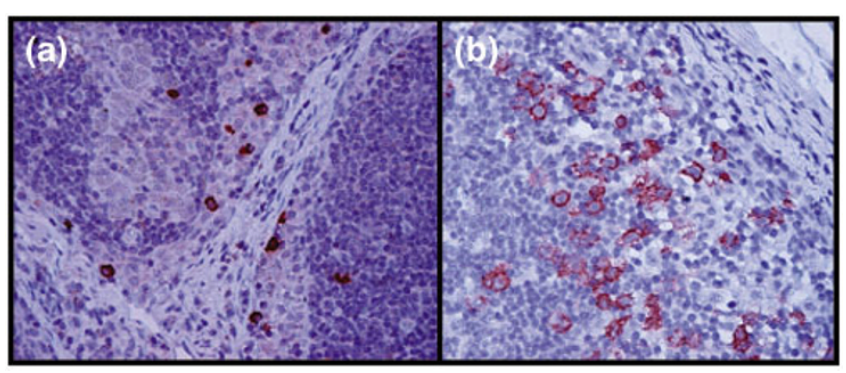

Expression of CD83 in a tumor-free sentinel lymph node (SLN) and of CD1a in a tumor-containing SLN from a breast cancer patient. Paraffinembedded (a) tumor-free or (b) tumor-containing SLN tissue was analyzed for expression of CD83 or CD1a, respectively, by immunohistochemistry. Typical examples of dendritic cell morphology stained with each antibody are shown (magnification, $\times 400$ ).

shown in Fig. 4. Tumor-free SLNs contained higher numbers of IL-10-producing cells $(P=0.02)$ and IL-12-producing cells $(P=0.12)$. Examination of sequential slides stained with anti-MHC class II or DC antibodies suggested that the cytokine-positive cells were MHC class II-positive and CD83-positive (Fig. 2). Tumor cells expressing IL-10 were not found in any of the SLNs examined.

\section{Comparison of SLN and lymph nodes draining from noncancer-containing breasts}

To understand whether the numbers of DCs seen in the SLNs were indicative of an activated state due to the presence of tumor, we examined 12 lymph nodes draining from the uninvolved noncancer-containing breast of patients with breast cancer (uninvolved lymph nodes) and compared them with the 50 SLNs from the breast cancer patients already analyzed. The numbers of CD1a-positive cells and CD83-positive cells were not significantly different when these uninvolved lymph nodes were compared with SLNs as a group (Fig. 5a,5b). In addition, when the number of CD83-positive cells in the tumor-free and tumorcontaining SLNs were compared with the number of CD83-positive cells in the uninvolved lymph nodes, the tumor-free SLNs were very similar to the uninvolved lymph nodes (Figs 3 and 5). When cytokine-expressing cells were examined, SLNs contained significantly higher numbers of both IL-10-expressing cells $(P=0.02)$ and IL-12-expressing cells $(P=0.002)$ than the uninvolved lymph nodes (Fig. $5 \mathrm{c}, 5 \mathrm{~d})$. Although the numbers of cytokine-expressing cells in both groups were low, the cytokine expressing cells were more abundant in the tumor-free SLNs.

\section{Discussion}

Our experiments were designed to determine whether the SLN was the site of T-cell activation in breast cancer patients. Identification of immunologically mature DCs within the SLN would support this hypothesis. In our exper-
Figure 2

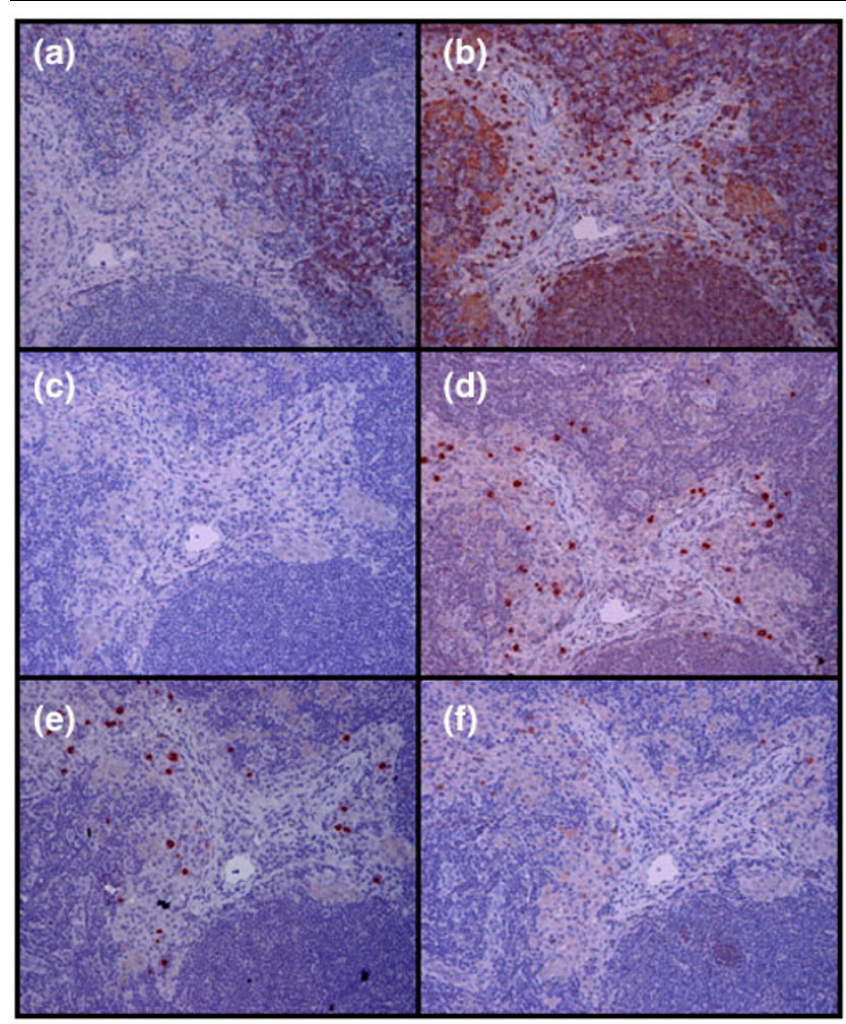

Expression of (a) CD3, (b) MHC class II, (c) CD1a, (d) CD83, (e) IL10 , and (f) IL-12 in a tumor-free sentinel lymph node (SLN) from a breast cancer patient analyzed by immunohistochemistry. Sequential sections from one block are shown (magnification, $\times 200$ ).

iments, mature DCs were defined by their expression of CD83, a marker that has been shown on DCs capable of antigen presentation and activation of naïv $T$ cells $[7,8]$. Our results show a definite trend toward higher numbers of CD83-positive DCs in tumor-free SLNs than in tumor-containing SLNs. In addition, tumor-free SLNs contained significantly higher numbers of IL-10-expressing cells. Both of these observations support the hypothesis that a tumorfree SLN is immunologically competent and is potentially a site of tumor-specific T-cell activation.

What remains unclear from our data is the type of immune response that occurs in the tumor-free SLNs. Identification of both IL-10-expressing and IL-12-expressing cells suggests two possibilities. The existence of IL-10 in tumors has been associated with immune suppression of a Th1 response and increased tumorigenicity [11]. But it has been shown convincingly through in vitro studies that IL-10 specifically affects immature DCs by inhibiting upregulation of MHC class II and costimulatory molecules and expression of CD83-positive cells. Mature CD83-expressing DCs are resistant to the effects of IL-10 [12]. Less information is available on the expression of IL-12 in tumor-draining lymph 
Figure 3
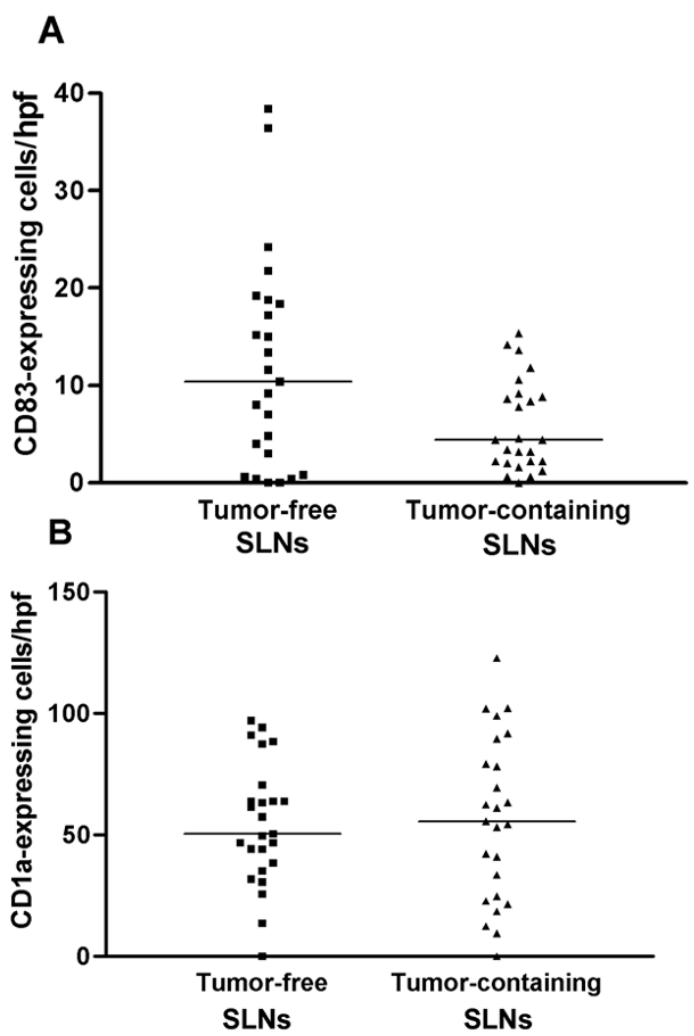

Comparison of the numbers of (a) CD83-expressing cells and (b) CD1a-expressing cells in tumor-free and tumor-containing sentinel lymph nodes (SLNs) of breast cancer patients. Each data point represents the mean number of cells per high-powered field (in five fields) (magnification, $\times 400$ ) from a paraffin-embedded section analyzed by immunohistochemistry. The horizontal bars represent the median number for each group. A trend toward a greater number of CD83expressing cells in the tumor-free SLNs than in the tumor-containing SLNs was seen $(P=0.07)$; no difference in the number of CD1aexpressing cells distinguished the two types of SLNs $(P=0.93)$.

nodes. Animal, as well as in vitro, studies support the role of IL-12 as a proinflammatory cytokine that drives DCs toward activation of Th1 [13,14]. Clearly, the existence of both of these two cytokines in the SLN and not in uninvolved lymph nodes supports the hypothesis that an active immune response is occurring in the SLNs. Future functional studies examining both DCs and T cells are needed to determine whether the SLN is a site of immune suppression, as suggested by the expression of IL-10, or of immune activation, as suggested by expression of IL-12.

Our results differ from those reported by Huang and colleagues [15], who compared S-100-positive DCs in SLNs and non-SLNs. Their conclusion, based on the morphology
Table 2

Comparison of CD83-expressing and CD1a-expressing dendritic cells in tumor-free and tumor-containing sentinel lymph nodes (SLNs) by tumor grades

\begin{tabular}{|c|c|c|c|}
\hline \multirow[t]{2}{*}{ Dendritic cells ${ }^{a}$} & \multicolumn{3}{|l|}{ Tumor grade $b$} \\
\hline & Grade I & Grade II & Grade III \\
\hline \multicolumn{4}{|c|}{ CD83-expressing cells } \\
\hline \multicolumn{4}{|l|}{ Tumor-free SLNs } \\
\hline Number & 3 & 11 & 8 \\
\hline Mean ( \pm SEM) & $4.5 \pm 4.5$ & $10.2 \pm 3.5$ & $13.0 \pm 2.9$ \\
\hline Median & 0 & 8 & 16.7 \\
\hline Range & $0-13.4$ & $0.4-38.4$ & $0.4-21.8$ \\
\hline \multicolumn{4}{|c|}{ Tumor-containing SLNs } \\
\hline Number & 3 & 12 & 8 \\
\hline Mean ( \pm SEM) & $9.1 \pm 2.7$ & $4.9 \pm 1.3$ & $5.8 \pm 1.8$ \\
\hline Median & 9.2 & 3.3 & 5.5 \\
\hline Range & $4.4-13.6$ & $0-14.2$ & $0.6-15.4$ \\
\hline$P$ value ${ }^{c}$ & 0.40 & 0.34 & 0.16 \\
\hline \multicolumn{4}{|c|}{ CD1a-expressing cells } \\
\hline \multicolumn{4}{|l|}{ Tumor-free SLNs } \\
\hline Number & 3 & 11 & 8 \\
\hline Mean ( \pm SEM) & $57.9 \pm 5.6$ & $60.0 \pm 10.5$ & $49.0 \pm 5.1$ \\
\hline Median & 63.2 & 63.5 & 48.6 \\
\hline Range & $46.8-63.8$ & $0-97.0$ & $25.6-70.6$ \\
\hline \multicolumn{4}{|c|}{ Tumor-containing SLNs } \\
\hline Number & 3 & 12 & 8 \\
\hline Mean $( \pm$ SEM) & $59.0 \pm 23.5$ & $48.7 \pm 8.5$ & $80.0 \pm 8.1$ \\
\hline Median & 53.2 & 41.6 & 74.3 \\
\hline Range & $21.6-102.2$ & $12.4-102.0$ & $54.4-122.8$ \\
\hline$P$ value & 1.0 & 0.41 & 0.01 \\
\hline
\end{tabular}

SEM, standard error of the mean. a Number of cells per highpowered field. $\mathrm{b}$ The grade was unknown for two tumors in the tumorcontaining SLN group and for three tumors in the tumor-free SLN group. ${ }^{\circ} P$ values determined by the Wilcoxon rank sum test comparing the number of CD83-expressing or CD1a-expressing dendritic cells in tumor-free SLNs versus tumor-containing SLNs for each tumor grade.

and density of the paracortical area and the number of paracortical S-100-positive DCs, was that the SLN was immunomodulated compared with the non-SLN. The SLNs examined in their study had reduced paracortical areas, reduced densities of paracortical DCs, and reduced frequencies of S-100-positive DCs with a predominance of immature, poorly dendritic DCs. Because they did not identify the tumor-free SLNs versus the tumor containing SLNs in their study, it is hard to compare our results with theirs. When we group our data and compare all SLNs with lymph 
Figure 4

(a)
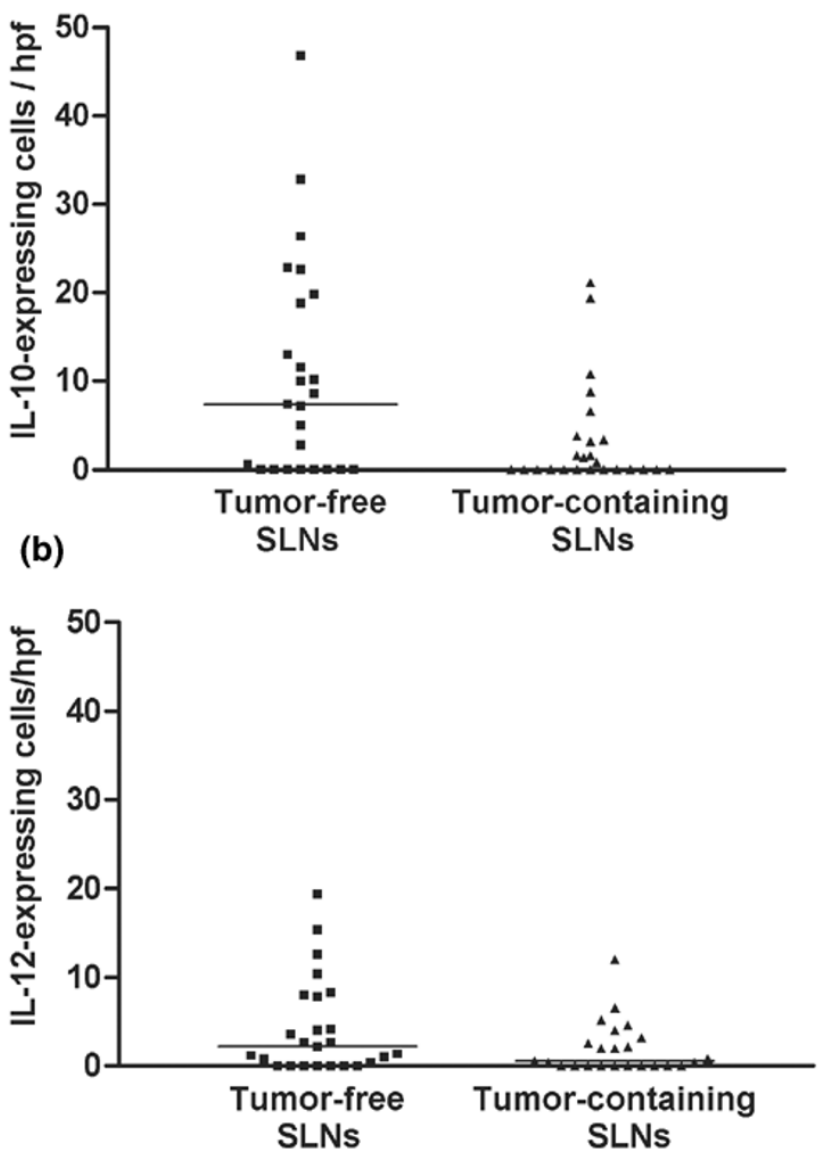

Comparison of the numbers of (a) IL-10-expressing cells and (b) IL-12expressing cells in tumor-free and tumor-containing sentinel lymph nodes (SLNs) of breast cancer patients. Each data point represents the mean number of cells per high-powered field (in five fields) (magnification, $\times 400$ ) from a paraffin-embedded section analyzed by immunohistochemistry. The horizontal bars represent the median number for each group. A significantly greater number of IL-10-expressing cells were seen in the tumor-free SLNs than in the tumor-containing SLNs (median number, 7.4 versus $0 ; P=0.02$ ); a trend toward a greater number of IL12 -expressing cells was seen in the tumor-free SLNs than the tumorcontaining SLNs (median number, 2.2 versus $0.5 ; P=0.12$ ).

nodes draining from the noncancer-containing breast of women with cancer, however, the numbers of DCs in the SLNs and these lymph nodes are not different, suggesting that the SLN is not immunomodulated compared with uninvolved nodes.

Few laboratories have examined the immune status of SLNs of human tumors [15-17]. Most work involving SLNs has been limited to studies of frozen or paraffin-embedded tissue. In one of the few studies in which fresh SLN tissue was available, an attempt was made to determine whether tumor-specific T cells could be categorized as Th1-type or Th2-type cells, based on cytokine profiles [16]. No classifi- cation was possible because the SLN T cells secreted both interferon gamma and IL-10 when stimulated with autologous tumor cells. However, these T cells were clearly tumor specific because they responded to autologous but not allogeneic tumor cells [16]. A more recent study examining the cytokines produced by Staphylococcal enterotoxin-Astimulated T cells from SLNs that drain primary melanoma showed both Th1 and Th2 cytokines could be measured by an enzyme-liked immunospot (ELISPOT) assay in tumorfree SLNs, whereas tumor-containing SLNs showed no increase in cytokine secretion [17]. The implication from this study was that immune responses were downregulated by micrometastases in the SLN. Both reports support our data and suggest that the SLN is the site of antigen-specific activation and that the tumor status of the node may predict the immune response to the tumor.

The pathologic features of the tumor may affect the immune response in the SLN. It is accepted that the tumor microenvironment affects the maturation state of the DCs in the tumor. Our data showed that the number of CD1a-expressing cells in the SLN was associated with tumor grade. When high-grade tumors (grade III) were analyzed, higher numbers of CD1a cells were found in the tumor-containing SLNs. This suggested that the undifferentiated state of the tumor inhibits DC maturation, causing an accumulation of immature DCs unable to activate naïve T cells and resulting in metastasis of the tumor to the draining node. A report by Vitale and colleagues [18] suggested that the lack of an immune response in the high-grade tumor is explained by the downregulation of MHC class I and TAP-1 and TAP-2 proteins in these tumors.

A recent report by Iwamoto and colleagues [9] examining CD83-expressing DCs in 130 human breast tumors demonstrated that the presence of tumor-infiltrating CD83expressing DCs correlated inversely with lymph node metastasis. Their data strongly suggested that CD83 DCs are involved in the initiation of the primary antitumor response. Our data, which showed a trend toward increased numbers of CD83-positive DCs in tumor-free SLNs compared with tumor-containing SLNs, further support this conclusion, and similarly suggest that the maturation state of the DCs predicts the metastatic potential of the tumor. Further experiments to compare the DCs in primary tumors and their SLNs are now underway in our laboratory to confirm the relationship between the maturation state of the DCs, the tumor grade, and the tumor metastatic potential.

\section{Conclusions}

In summary, we found a trend toward higher numbers of CD83-positive DCs in tumor-free SLNs than in tumor-containing SLNs. In addition, tumor-free SLNs contain significantly higher numbers of IL-10-expressing cells. Both of 
Figure 5

(a)

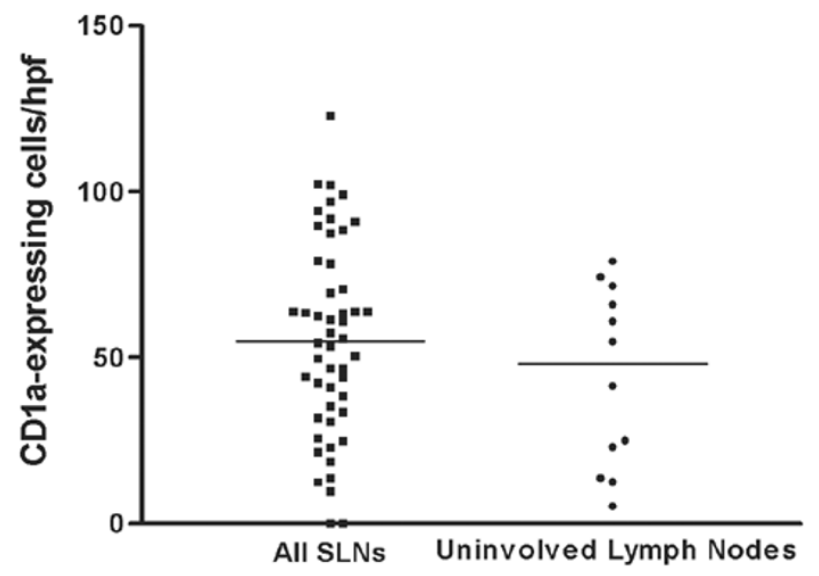

(c)

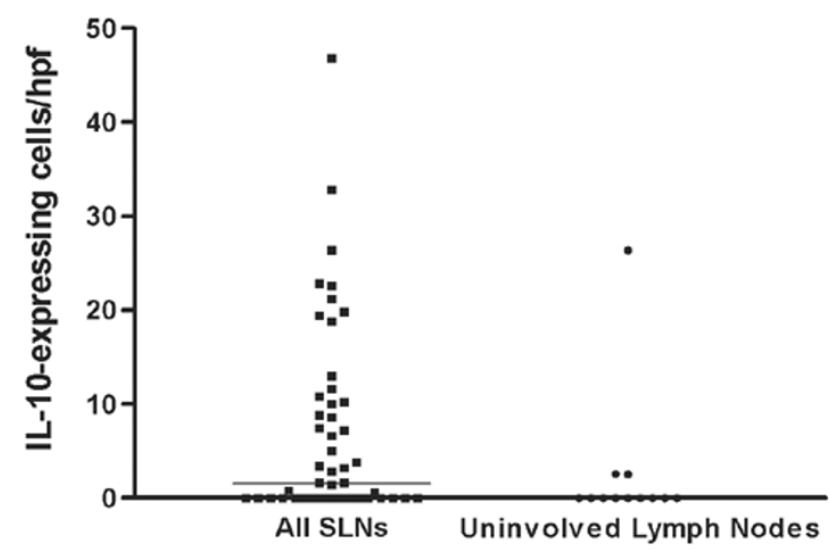

(b)

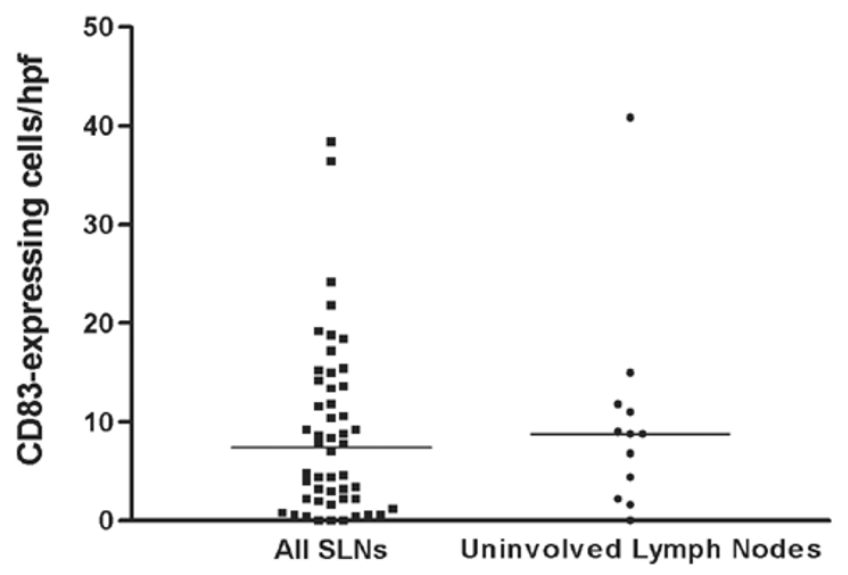

(d)

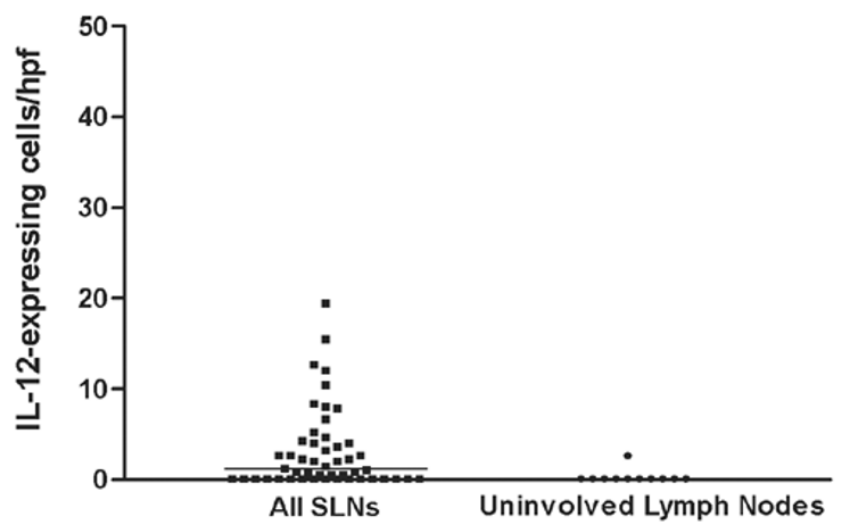

Comparison of (a) CD1a-expressing cells, (b) CD83-expressing cells, (c) IL-10-expressing cells and (d) IL-12-expressing cells in sentinel lymph nodes (SLNs) of study patients and lymph nodes draining from noncancer-containing breasts (uninvolved lymph nodes) of women with breast cancer. Each data point represents the mean number of cells per high-powered field (in five fields) (magnification, $\times 400$ ) from a paraffin-embedded section analyzed by immunohistochemistry. The horizontal bars represent the median number for each group. The numbers of CD83-expressing cells or CD1a-expressing cells in SLNs were not different from those in the uninvolved lymph nodes, but a significant difference was seen when expression of IL-10 or IL-12 was examined. Significantly greater numbers of IL-10-expressing and IL-12-expressing cells were seen in the SLNs compared with the uninvolved lymph nodes $(P=0.02$ and $P=0.002$, respectively).

these observations support the hypothesis that the tumorfree SLN is immunologically competent and is potentially a site of tumor-specific T-cell activation. To establish the prognostic potential of the DCs in the SLN, we are now undertaking functional studies to compare the immune status of DCs isolated from the primary tumors of patients with tumor-free SLNs and those with tumor-containing SLNs.

\section{Competing interests}

None declared.

\section{Acknowledgements}

The authors thank Sandra Kinney for excellent technical assistance. In addition, they are grateful for the technical assistance provided by the staff of the Breast Tumor Bank at The University of Texas MD Anderson Cancer Center. The Breast Tumor Bank is supported through a grant from The Nellie B Connally Breast Cancer Research Fund. Statistical analysis was performed with the help of Marcella Johnson, of the Department of Biostatistics, MD Anderson Cancer Center. This research was supported by Department of the Army Grant DAMD17-00-1-0680 (to NJP) and by funds from the University Cancer Foundation of the University of Texas MD Anderson Cancer Center (to NJP).

\section{References}

1. Banchereau J, Steinman RM: Dendritic cells and the control of immunity. Nature 1998, 392:245-252.

2. Bell D, Chomarat P, Broyles D, Netto G, Harb GM, Lebecque $S$, Valladeau J, Davoust J, Palucka KA, Banchereau J: In breast carcinoma tissue, immature dendritic cells reside within the tumor, whereas mature dendritic cells are located in peritumoral areas. J Exp Med 1999, 190:1417-1425. 
3. Cella M, Sallusto F, Lanzavecchia A: Origin, maturation and antigen presenting function of dendritic cells. Curr Opin Immunol 1997, 9:10-16.

4. Lapointe R, Toso JF, Butts C, Young HA, Hwu P: Human dendritic cells require multiple activation signals for the efficient generation of tumor antigen-specific T lymphocytes. Eur J Immunol 2000, 30:3291-3298.

5. Giuliano $A E$, Jones RC, Brennan M, Statman R: Sentinel lymphadenectomy in breast cancer. J Clin Oncol 1997, 15:2345-2350.

6. Leong SPL: Paradigm of metastasis for melanoma and breast cancer based on the sentinel lymph node experience. Ann Surg Oncol 2004, 11:192S-197S.

7. Zhou LJ, Schwarting R, Smith HM, Tedder TF: A novel cell-surface molecule expressed by human interdigitating reticulum cells, Langerhans cells, and activated lymphocytes is a new member of the Ig superfamily. J Immunol 1992, 149:735-742.

8. Zhou LJ, Tedder TF: CD14+ blood monocytes can differentiate into functionally mature $\mathrm{CD}^{2} 3^{+}$dendritic cells. Proc Natl Acad Sci USA 1995, 93:2588-2592.

9. Iwamota $\mathrm{M}$, Shinohara $\mathrm{H}$, Miyamoto A, Okuzawa M, Mabuchi $\mathrm{H}$ Nohara H, Gon G, Toyoda M, Tanigawa N: Prognostic value of tumor-infiltrating dendritic cells expressing CD83 in human breast carcinomas. Int J Cancer 2003, 104:92-97.

10. Kalinski P, Hilkens CMU, Wierenga EA, Kapsenberg ML: T-cell priming by type- 1 and type- 2 polarized dendritic cells: the concept of a third signal. Immunol Today 1999, 20:561-567.

11. Halak BK, Maguire HC, Lattime EC: Tumor-induced interleukin10 inhibits type 1 immune responses directed at a tumor antigen as well as a non-tumor antigen present at the tumor site. Cancer Res 1999, 59:911-917.

12. Jonuleit $H$, Schmitt E, Steinbrink K, Enk AH: Dendritic cells as a tool to induce anergic and regulatory $\mathrm{T}$ cells. Trends Immunol 2001, 22:394-400.

13. Heufler C, Koch F, Stanzl U, Topar G, Wysocka M, Trinchieri G, Enk A, Steinman RM, Romani N, Schuler G: Interleukin-12 is produced by dendritic cells and mediates T helper 1 development as well as interferon-gamma production by $\mathrm{T}$ helper 1 cells. Eur J Immunol 1996, 26:659-668.

14. Cella $M$, Scheidegger $D$, Palmer-Lehmann $K$, Lane $P$, Lanzavecchia A, Alber G: Ligation of CD40 on dendritic cells triggers production of high levels of interleukin-12 and enhances $T$ cell stimulatory capacity: T-T help via APC activation. J Exp Med 1996, 184:741-747.

15. Huang RR, Wen D-R, Guo J, Giuliana AE, Nguyen M, Offodile R, Stern S, Turner R, Cochran AJ: Selective modulation of paracortical dendritic cells and T-lymphocytes in breast sentinel lymph nodes. Breast 2000, 6:225-232.

16. Chu Y, Hu HM, Winter H, Wood WJ, Doran T, Lashley D, Bashey J, Schuster J, Wood J, Lowe BA, Vetto JT, Weinberg AD, Puri R, Smith JW, Urba WJ, Fox BA: Examining the immune response in sentinel lymph nodes of mice and men. Eur J Nucl Med 1999, 26(Suppl):S50-S53.

17. Leong SPL, Peng M, Zhou Y-M, Vaquerano JE, Chang JWC: Cytokine profiles of sentinel lymph nodes draining the primary melanoma. Ann Surg Oncol 2002, 9:82-87.

18. Vitale M, Rezzani R, Rodella L, Zauli G, Grigolato P, Cadei M, Hicklin DJ, Ferrone S: HLA class I antigen and transporter associated with antigen processing (TAP1 and TAP2) downregulation in high-grade primary breast carcinoma lesions. Cancer Res 1998, 58:737-742. 